French reference centres experience. Eur J Hum Genet. 2018;26: 960-71.

2. Batu ED, Karadag O, Taskiran EZ, et al. A Case series of adenosine deaminase 2-deficient patients emphasizing treatment and genotype-phenotype correlations. J Rheumatol. 2015;42: 1532-4.

3. Zhou Q, Yang D, Ombrello AK, et al. Early-onset stroke and vasculopathy associated with mutations in ADA2. N Eng J Med. 2014;370:911-20.

4. Taskiran EZ, Sonmez HE, Ayvaz DC, et al. Hypomorphic RAG1 defect in a child presented with pulmonary hemorrhage and digital necrosis. Clin Immunol. 2018;187:92-4.

5. Navon Elkan P, Pierce SB, Segel R, et al. Mutant adenosine deaminase 2 in a polyarteritis nodosa vasculopathy. N Eng J Med. 2014;370:921-31.
6. Berkun Y, Segel R, Navon-Elkan P. Adenosine deaminase 2 deficiency: more than monogenic vasculitis. Isr Med Assoc J. 2017;19:435-7.

7. van Montfrans J, Zavialov A, Zhou Q. Mutant ADA2 in vasculopathies. N Eng. J Med. 2014;371:478.

8. Ben-Ami T, Revel-Vilk S, Brooks R, et al. Extending the clinical phenotype of adenosine deaminase 2 deficiency. J Pediatr. 2016;177:316-20.

9. Hashem H, Egler R, Dalal J. Refractory pure red cell aplasia manifesting as deficiency of adenosine deaminase 2. J Pediatr Hematol Oncol. 2017;39:293-6.

10. Schepp J, Proietti M, Frede N, et al. Screening of 181 patients with antibody deficiency for deficiency of adenosine deaminase 2 sheds new light on the disease in adulthood. Arthritis Rheumatol. 2017;69:1689-700.

\title{
Reply to Sönmez et al.
}

\author{
Mélanie Rama ${ }^{1}$ - Isabelle Touitou $\mathbb{1}^{1,2} \cdot$ Guillaume Sarrabay ${ }^{1,2}$
}

Received: 12 July 2018 / Accepted: 19 July 2018 / Published online: 11 September 2018

(c) European Society of Human Genetics 2018

We thank Sönmez et al. for their comment on our paper providing a provisional decision tree for the genetic diagnosis of deficiency of adenosine deaminase 2 (DADA2). This autosomal recessive disorder was primarily described as inflammatory vasculitis [1, 2]. More recently, other phenotypes have been described, such as pure haematological presentation (aplastic anaemia, erythroblastopenia, etc), without vascular or inflammation features [3-6].

Gene sequencing can be performed by either Sanger or NGS (Next Generation Sequencing) technique. In our original article, Rama et al. proposed a decision tree to help physicians prioritise molecular screening among SAID patients with possible DADA2 and benefit from a fast diagnosis with Sanger analysis [7]. Patients who do not meet the prerequisites have less probability to have DADA2. However, lack of some of the prerequisites does

Guillaume Sarrabay

guillaume.sarrabay@inserm.fr

1 Laboratory of Rare and Autoinflammatory Genetic Diseases and CEREMAIA, Montpellier University Hospital, Montpellier, France

2 Genetics \& Immunopathology of Inflammatory Osteoarticular Diseases, IRMB, Univ Montpellier, INSERM, Montpellier, France not exclude the diagnosis of DADA2, which can be made using NGS strategy.

Sönmez et al. have tested the proposed decision tree on ten genetically confirmed DADA2 patients and eight periarteritis nodosa (PAN) patients. All but one met the prerequisites: a true DADA2 patient with only haematological presentation who was not eligible for Sanger sequencing using the original tree. These authors pinpoint that atypical presentations such as aplastic anaemia are missed by our provisional decision tree. We concede that it might be relevant to implement the haematological features as an alternative prerequisite.

In the initial paper by Navon Elkan et al., CECRI/ADA2 was discovered in a cohort of PAN patients sequenced by WES, supporting that PAN and DADA2 may be undistinguishable based on clinical features only prior to genetic analysis [1]. Therefore, it is not surprising that all of their PAN patients meet the prerequisites. True PAN patients are not disadvantaged by having Sanger sequencing. The authors had a high prevalence of DADA2 consanguineous patients (9/10 patients). Consanguinity is common in the Turkish population, but rare in the European population and in our confirmed DADA2 patients (2/11 families) [7]. Hence, we believe that in most populations, consanguinity is not relevant as a prerequisite for Sanger analysis.

In conclusion, Sönmez et al. have highlighted the importance of the pure haematological presentation. They 
propose to implement this feature in the diagnostic decision, and we agree with that. However, consanguinity should be considered in specific populations, but cannot be universally used in our opinion.

\section{Compliance with ethical standards}

Conflict of interest The authors declare that they have no conflict of interest.

\section{References}

1. Navon Elkan P, Pierce SB, Segel R, Walsh T, Barash J, Padeh S, et al. Mutant adenosine deaminase 2 in a polyarteritis nodosa vasculopathy. N Engl J Med. 2014;370:921-31.
2. Zhou Q, Yang D, Ombrello AK, Zavialov AV, Toro C, Zavialov $\mathrm{AV}$, et al. Early-onset stroke and vasculopathy associated with mutations in ADA2. N Engl J Med. 2014;370:911-20.

3. Hashem H, Egler R, Dalal J. Refractory pure red cell aplasia manifesting as deficiency of adenosine deaminase 2. J Pediatr Hematol Oncol. 2017;39:e293-6.

4. van Montfrans J, Zavialov A, Zhou Q. Mutant ADA2 in vasculopathies. N Engl J Med. 2014;371:478.

5. Ben-Ami T, Revel-Vilk S, Brooks R, Shaag A, Hershfield MS, Kelly SJ, et al. Extending the clinical phenotype of adenosine deaminase 2 deficiency. J Pediatr. 2016;177:316-20.

6. Berkun Y, Segel R, Navon-Elkan P. Adenosine deaminase 2 deficiency: more than monogenic vasculitis. Isr Med Assoc J. 2017;19:435-7.

7. Rama M, Duflos C, Melki I, Bessis D, Bonhomme A, Martin H, et al. A decision tree for the genetic diagnosis of deficiency of adenosine deaminase 2 (DADA2): a French reference centres experience. Eur J Hum Genet. 2018;26:960-71. 